\title{
Pedagogical Assumptions Via the Internet Culture
}

\author{
Eranda Bilali (Halluni), Ph. D \\ erandabilali@yahoo.com \\ University of Shkodra "Luigj Gurakuqi" \\ Fatmir Vadohej, Assoc. Prof. Dr. \\ fatmirvadahi@gmail.com \\ University of Shkodra "Luigj Gurakuqi" \\ Mehdi Kroni, Ph. D \\ kronimehdi@yahoo.com \\ University of Shkodra "Luigj Gurakuqi" \\ Edit Lezha, Ph. D (c) \\ editlezha@gmail.com \\ University of Shkodra "Luigj Gurakuqi”
}

\begin{abstract}
The pervasion of digital technology through our lives is part of a broader set of phenomena. More and more we speak now of the network society, knowledge and learning. In fact, the networks have come into our lives, permeating our daily relations with their presence. New integrations have risen between men and networks capable to create the evolution, to change the forms of our knowledge into new learning contexts. Technology makes grading easier, lesson planning easier, provides access to additional information and resources, saves time, and helps the learner expand his or her learning opportunity beyond the classroom walls (Technology in Education Consortium, 2014; Nye \& McConrville, 2007). In such a learning community, teachers and students in the role of diversity, skills and participatory methods, collaborate on the construction and reconstruction of knowledge as well as the exploitation of the sense of life experiences. The interaction that the teacher assumes is the function of priority organizer of educational environments with an appropriate learning and a conscious participation. These concepts shape the basis of treatment of the paper for the presenting, authors together with their vision and generalize from contemporary literature that emphazise the role of internet culture in education.
\end{abstract}

Keywords: internet, society, culture, pedagogy, education

\section{Introduction}

At school students have central position, stimulated and encouraged in their conscious participation in the various learning experiences in both individual and collaborative forms, where the attitude of active research in the acquisition of knowledge, promotes the need to find personal interpretation to common group learning. The aim of global growth projects (cognitive, affective, social, etc. ) is having free and responsible persons, capable of processing a subjective identity and participating in a critical and active life in society; in learning situations subjects are different genders, ages, rhythms and types of development, social classes, religions, cultures, countries of origin and experience of social life where such differences, 
becoming a resource that offers a significant contribution to the growth of the whole the educational community. In the field of education, advances in technology offer many benefits. Schools and individual teachers find it easier to communicate with parents and the community. Technology makes grading easier, lesson planning easier, provides access to additional information and resources, saves time, and helps the learner expand his or her learning opportunity beyond the classroom walls (Technology in Education Consortium, 2014; Nye \& McConrville, 2007).

\section{Internet culture and pedagogy}

According to a starting point of Kantian philosophy, the man reaches humanity only through education, where the task of the educator should be to find out what the interest of a child is and help him fulfil it, but the teachers are not always available to sacrifice their role, down among the students, to find new ideas and solutions. Effective communication is the essential element to successful online experiences (Cavanaugh \& Hargis, 2010; Duncan \& Barnett, 2009; Garrison \& Vaughan, 2008; Yang et al., 2010). The online environment offers unique opportunities for rich dialogue and interaction as well as the possibility of promoting cognitive engagement with deep educational meaning (Garrison \& Clevelandlnnes, 2005).

The real difficulty is something else, failing to put students in touch with the object of study, thus allowing them to make "experience", difficulty that resulted in the reorganization of school learning "symbolic -reconstructive" based on language and written text. Could the use of the media and, above all, the

Introduction of new media, enable the school to regain control of the experiential method to activate the process of teaching and learning aims? And yet, the technology can effectively penetrate in everyday teaching to the solution of various problematic? Constructivist theory suggests that effective online learning is active, not passive; allows learners a level of control over learning experiences; encourages collaboration and cooperation among learners; and promotes interaction and social presence. While there is no single educational theory specific to online learning, the framework for a model has been developed from these cognitive and constructivist theories: the Community of Inquiry (Col) Model (Garrison, Anderson, \& Archer, 2000). A community of inquiry is a group of students and at least one instructor who engage in discussions focused on constructing knowledge or solving a problem (Garrison et al., 2000). The Col model assumes that the best learning experience is - a collaborative communication process for the purpose of constructing meaningful and worthwhile knowledgell (Garrison et al., 2000, p. 92). The key distinction in this type of education is that it is institutionfocused, faculty-centered, and delivered primarily through electronic media to remote or distant sites (Gibbs, 2000). It is normal to think that talking about technologies applied to teaching, for conveying a simpler and conscious knowledge, is something innovative and unprecedented but it is not: the close relations that interconnect between these two sectors stem from the simple fact that the educational act is in itself a relational and communicative ergo "act", any innovation in the field of communication allows a positive impact not only in the sphere of education, but also on how to exercise the same methodology and didactics.

\section{Learning with the network in education settings}

More and more we speak now of the network society, knowledge and learning. In fact, the networks have come into our lives, permeating our daily relations with their presence. New integrations have risen between men and networks capable to create the evolution, to change the forms of our knowledge into new learning contexts. In this landscape, the society becomes the context of a central research for a significant learning, through new forms of sharing and distribution, leaving apart the traditional limits of space and time. New references for learning through the international reflection and expressions such as lifelong learning and distance learning where, the expression "You never stop learning" adequately expresses a profound truth: whether it could change form and content, to adapt to new learning demands and needs of the adult life, but the need and willingness to learn does not end over the time. Waits and Lewis (2003) provide an alternative view, as they consider distance education to be an entity distinct from distance learning. They suggest that distance education is "education or training courses delivered to remote (off-campus) sites via audio, video (live or prerecorded), or computer technologies, including both synchronous (i. e., simultaneous) and asynchronous (i. e., not simultaneous) instruction"). Keegan (1996) also views distance education as being institution-focused educational experiences that are provided to students at sites separated from the instructors. The pivotal elements of these definitions include the belief that the institution dictates the content, context, and time of the learning opportunity with the student adhering to the learning opportunity restrictions driven by the institutional focus. 
It is on these ideas that we have developed and tried to focus on the dynamics of e-learning promoted and enhanced by resorting to the use of increasingly sophisticated technologies, such as forms of optimal learning thanks to which, today, each of us can keep alive their education throughout their lives. In their lives outside the confines of the school environment, young students are immersed in a wide range of digital interactions. When they enter the world of school, students often find themselves in an environment that largely discourages, if not prohibits, the use of the same digital technologies that engage them as learners outside of school. Worldwide the interest of trainers and teachers is focused on the Internet, which is proved to be an environment where it is possible to teach and learn in new, wider and flexible ways, with positive implications on the organizational and economic area. The information found by this form of learning is supported by new technologies and highlights the importance played from e-learning in the world of education at any level or age. The environments nowadays can make use of two powerful extraordinary challenges with the intent of a major innovation in their professional training: 1. the strategic need associated with the technological opportunity that is the strategic need of enhancement of the most important competitive factor and 2. capital human and its constant development in a turbulent mutational environment and the opportunity offered by the technologies of network communication.

Regarding the first aspect, the need to have, to attract and use the best human resources, as a quality factor in their different performance and therefore to achieve lasting competitive advantages, leads to the need of developing methods and systems of continuous training and Tapping the potential of the people employed. Integrated energy and resources, are in the best position to communicate, and to reset the times related to the limits of space and time where dynamic was established and articulated in being able to make out the best collective potential. This is one of the areas for reflection that permeates our current society.

The recent crucial turning point starts with the transition to online education since the advent of the Internet has increased to a new philosophy of education, based more on the reuse and sharing of knowledge already possessed by the participants rather than the transmission between teacher and learner. Ramig (2009) expanded on the use of blogs for educational purposes, claiming that social networking sites, such as Twitter, Facebook, and MySpace, also established a presence in class and in the lives of students of all ages. This form of social media can also cultivate communication by extending classroom discussions outside of class time and allowing students to have control over their learning environment (Ramig, 2009).. Ramig (2009) recommended that teachers not only become familiar with the online tools to incorporate proper safety measures in class, but also advised teachers to make extra efforts to use these tools as part of the educational process.

The starting point of this reflection can be doubtful related to the current problem of distance learning and in particular of online education: what is the relationship between quality and interactivity? From the point of view of those who design an online course content and interactivity can be integrated, and the financial implications, methodological and didactic choice (about the weight to be attributed more to one or other possibilities) are very high. The online courses are differentiated between web-based courses (based on materials) and based systems Computer Conferencing courses (based more on the interaction between people), while the course-interactivity formed at a distance can be three types: with the teachers, with the materials and among the students.

When designing a course, it is very important to consider what and how the students interact, because if the interaction between the participants has a relevant cost, it is quite true, that interactivity is more difficult without providing an initial meeting clarifying on costs, particularly if participants come from distant locations. From the methodological point of view the mixed strategy is preferable, through a meeting of all course participants, especially when it comes to planning activities, establishing roles or subdivisions of tasks, even if it is not always feasible, and requires a level of expenditure proportional because of the distance.

In such a learning community, teachers and students in the role of diversity, skills and participatory methods, collaborate on the construction and reconstruction of knowledge as well as the exploitation of the sense of life experiences. The interaction that the teacher assumes is the function of priority organizer of educational environments with an appropriate learning and a conscious participation. The teacher is a director involved in motivating the potential of each student in the process of acquisition of skills and competencies. Motivation to learn, after all, is not homogeneous or un modifiable in the same person, since the individual is the result of individual factors, social and cultural rights, will be more influenced by the same learning itself, but more balanced and stronger will be the practice of the process itself activated. 
Innovate means not filing the application for equipment to be put at the service of the things that you already do to keep them in the same way, since innovation is not the service that technology makes to insiders but the service itself, that is, head of who is providing it; it follows that attention should be paid to training for those who, in purely educational field, are likely to become "obsolete" in spite of the qualifications possessed. The new generations have an underlying need: to be educated to the management and search of knowledge, understood as communication with the new media, in order to gain the knowledge that citizens can know how to consume the use of the media themselves; thus reinterpreted, the reality technology to be used directly as a real asset in the process of traditional teaching, creating not only innovative models but those models based on collaborative and bidirectional communication processes, as well as outlined in recent years. If innovation initiated the transition from the "information society", understood as mass information with the distribution of default data and standardized to a "knowledge society", understood as cognitive participation of every individual to the wealth of knowledge through the skills possessed, Media Education focuses on the way in which the subject form his personality in a "media" society, questioning the way to safeguard the development of the individual, protecting its autonomy and creative freedom, focusing on ways fruition, interaction and importance of the understanding of "what and how" they can change attitudes and behavior in the new generations.

\section{Conclusions}

New criteria should be established to be able to make choices about which role and for which purposes internet culture can be used with a priority school context. The reading of the " medium", considered as construction, put on stage and represented from the reality, where the perceptual experience is determined and conditioned mainly by the relationship of analogy of the image has with what is still more effective "since writing moving", will pretend accuracy and objectivity: the media is not the reality, but the mere representation of moving it. In digital-age learning cultures, modern technology allows learning to be portable, constant, and interactive. It provides learners with an expanded audience who can provide global perspectives (McKnight, 2014). As digital-age learning is ever evolving, the educators' interconnectivity with it becomes increasingly more important, at least according to the article, Learning and Leading with Technology (McKnight, 2014). From this reading it is clear the role of the teacher in leading the students to mature critical awareness of choices and strategies that regulate and underlie communication, which is the source of man's look on man in its modernity. Moreover, it is clear how technology provides an opportunity enabling schools to reconfigure forms distributed differently for an additional didactic "differentially effective" for the construction of integrated self-learning environments able to expand on the basis of experience that the students perform.

\section{References}

Cavanaugh, C., \& Hargis, J. (2010). Redefining school from site to service: Learning in and from K-12 online education. Distance Learning, 7(2), 1-5.

Garrison, D. R., Anderson, T., \& Archer, W. (2000). Critical inquiry in a text-based environment: Computer conferencing in higher education. The Internet and Higher Education, 2(2-3), 87-105. Retrieved from http://communitiesofinquiry. com

Garrison, D. R., \& Cleveland-Innes, M. (2005). Facilitating cognitive presence in online learning: Interaction is not enough. The American Journal of Distance Education, 19(3), 133-148.

Gibbs (2000, May). Distance education: Guidelines for good practice. Report to the American Federation of Teachers Higher Education Department. Washington DC [Electronic version].

Gilles Deleuze and Claire Parnet, Dialogues (London, 1977), pp. 126-7.

Keegan, D. (1996). Foundations of distance education. Routledge Studies in Distance Education. (3rd Ed. ). London and New York: Routledge.

McKnight, K. (2014). Top 12 ways technology changed learning. Retrieved from http://www. teachhub. com/howtechnology-changed-learning 
Nye, D., \& McConville, L. (2007). The bridging influence of technology and collaboration. Delta Kappa Gamma Bulletin, 74(2), 5-7.

Ramig, R. (2009). Social media in the classroom. Multimedia \& Internet@Schools, 16(6), 8.

Waits, T. \& Lewis, L. (2003) Distance education at degree-granting postsecondary institutions: 2000-2001, US Department of Education, National Center for Education Statistics (NCES 2003-017).

https://en. wikiversity. org/wiki/Digital_culture 